\title{
Soft x-ray seeding studies for the SLAC Linac Coherent Light Source II
}

\author{
E. Hemsing, ${ }^{*}$ G. Marcus, ${ }^{\dagger}$ W. M. Fawley $\odot,{ }^{\ddagger}$ R. W. Schoenlein,${ }^{\S}$ R. Coffee, \\ G. Dakovski, J. Hastings, Z. Huang, D. Ratner, and T. Raubenheimer \\ SLAC National Accelerator Laboratory, Menlo Park, California 94025, USA \\ G. Penn" \\ Lawrence Berkeley National Accelerator Laboratory, Berkeley, California 94720, USA
}

(Received 18 July 2019; published 4 November 2019)

\begin{abstract}
We present the results from studies of soft $\mathrm{x}$-ray seeding options for the LCLS-II $\mathrm{x}$-ray free electron laser (FEL) at SLAC. The LCLS-II will use superconducting accelerator technology to produce x-ray pulses at up to $1 \mathrm{MHz}$ repetition rate using $4 \mathrm{GeV}$ electron beams. If properly seeded, these pulses will be nearly fully coherent, and highly stable in photon energy, bandwidth, and intensity, thus enabling unique experiments with intense high-resolution soft x-rays. Given the expected electron beam parameters from start to end simulations and predicted FEL performance, our studies reveal echo enabled harmonic generation (EEHG) and soft x-ray self-seeding (SXRSS) as promising and complementary seeding methods. We find that SXRSS has the advantage of simplicity and will deliver 5-35 times higher spectral brightness than EEHG in the 1-2 nm range, but lacks some of the potential for phase-stable multipulse and multicolor FEL operations enabled by external laser seeding with EEHG.
\end{abstract}

DOI: 10.1103/PhysRevAccelBeams.22.110701

\section{INTRODUCTION}

Free electron laser (FEL) seeding offers the potential to produce bright, nearly Fourier-transform limited (FTL), and highly repeatable pulses compared to pulses produced by self-amplified spontaneous emission (SASE). The general motivation for FEL seeding arises from the need for control over the longitudinal coherence (i.e., spectral amplitude and phase) of $\mathrm{x}$-ray pulses to enable a broad range of science applications. In the soft x-ray (SXR) range of $E_{\mathrm{ph}}=280 \mathrm{eV}(4.4 \mathrm{~nm})$ to $1.2 \mathrm{keV}(1 \mathrm{~nm})$, defined by the absorption edges of various earth-abundant elements (for example, C (284 eV), N (410 eV), O (543 eV), Fe $(707 \mathrm{eV}), \mathrm{Cu}(933 \mathrm{eV})$, and $\mathrm{Zn}(1,022 \mathrm{eV}))$, time-resolved $\mathrm{x}$-ray spectroscopy and time-resolved resonant $\mathrm{x}$-ray scattering are powerful probes of excited-state dynamics in material and molecular complexes. The scientific impact of these methods relies on simultaneously optimizing both the pulse length and the bandwidth for specific science

\footnotetext{
*ehemsing@slac.stanford.edu gmarcus@slac.stanford.edu

fawley@slac.stanford.edu

\$rwschoen@slac.stanford.edu

gepenn@lbl.gov
}

Published by the American Physical Society under the terms of the Creative Commons Attribution 4.0 International license. Further distribution of this work must maintain attribution to the author(s) and the published article's title, journal citation, and DOI. requirements, including the ability to trade-off time resolution against spectral resolution at close to the Fourier transform limit.

The range of interest for the majority of science applications extends from high time resolution 10 fs (180 meV bandwidth) to high spectral resolution at $60 \mathrm{fs}(30 \mathrm{meV})$. The $10 \mathrm{fs}$ timescale is sufficient to resolve bond breaking and formation processes, vibrational dynamics, and most charge transfer processes. At the same time, a spectral resolution of $180 \mathrm{meV}$ is sufficient to resolve relevant near-edge features (e.g., for absorption spectroscopy or resonant scattering) which are defined by core-hole lifetime broadening, typically $>200 \mathrm{meV}$. Alternatively, high energy resolution at the $20-30 \mathrm{meV}$ scale can resolve a wide range of low energy collective modes in correlated materials (e.g., the typical energy gap for unconventional superconductors is $\sim 50 \mathrm{meV}$ ), and can further resolve many relevant vibrational modes (e.g., metal-ligand stretch) in molecules. Simultaneous time resolution at the $60 \mathrm{fs}$ scale enables one to follow the evolution of collective modes in response to specific external perturbations (e.g., resonant vibrational excitation of materials or chargetransfer excitations in molecules). More detail on the specific science targets of interest can be found in $[1,2]$.

Within this range of interest there are important potential practical benefits that arise from seeding when compared with stochastically noisy SASE, which has a full bandwidth of $\sim 1-2 \mathrm{eV}$ at SXR spectral energies (i) Higher average spectral flux (photons/sec/meV). A seeded XFEL delivers most of the power in the spectral bandwidth of interest, 


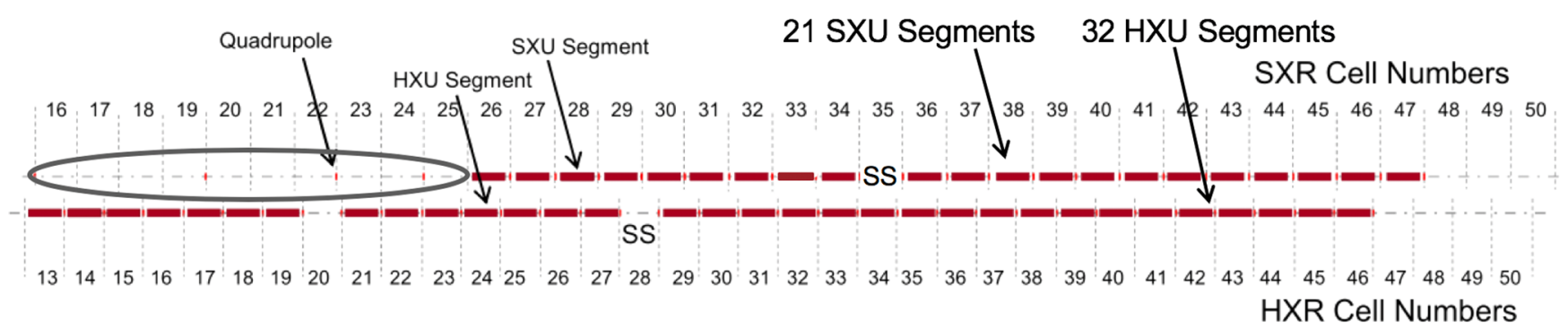

FIG. 1. Layout of the LCLS-II soft X-ray (top) and hard X-ray (bottom) undulators. Both undulator lines will be fed by the $1 \mathrm{MHz}$ e-beam, which goes left to right. The SXR line is composed of 21 undulators segments each $3.4 \mathrm{~m}$ in length, occupying a total footprint of $96 \mathrm{~m}$. General e-beam and undulator properties are given in Tables I and II. There is about $40 \mathrm{~m}$ of space available upstream of the SXR line (circled) that could be used for external seeding infrastructure. Slot 35 (undulator no. 10) will accommodate the SXRSS system.

therefore increasing the brightness (photons $/ \mathrm{meV}$ ) and minimizing any losses from post-XFEL spectral filtering, which can be significant for SXR optics [3,4]. (ii) Timing synchronization from external seeding with short laser pulses (e.g., pump/probe). This enables better performance (e.g., time resolution) and reduces the need for timestamping and pulse-sorting by reducing electron timing jitter to the few-femtosecond level or below $[5,6]$. (iii) Improved amplitude stability (particularly with external seeding) compared with a SASE XFEL filtered with a monochromator [7,8]. (iv) Coherent control of the central wavelength within the SASE bandwidth and control of the coherent relative bandwidth down to the $10^{-4}$ level $[9,10]$. (v) Coherent two-pulse and multicolor, phase-locked operations (e.g., [11]). These structures in the photon pulse can induce specific low-energy transitions in electrons, or target distinct atoms with controlled relative timing.

Here we report on studies of seeding options for $1.2 \mathrm{keV}$ $(1 \mathrm{~nm})$ and $620 \mathrm{eV}(2 \mathrm{~nm})$ soft x-rays at LCLS-II. Results suggest that the best overall FEL performance and flexibility is provided by a combination of soft x-ray selfseeding (SXRSS) and echo enabled harmonic generation (EEHG). This conclusion is driven by the need to address key SXR science targets and the concomitant requirements for FEL spectral brightness, spectral purity, bandwidth, stability, and tunability. Self-seeding relies on monochromatization and amplification of a SASE pulse produced by the upstream portion of the FEL, and has successfully been implemented at both hard and soft x-ray energies at LCLS $[3,12]$. EEHG $[13,14]$ has recently been demonstrated with high-gain up to $211 \mathrm{eV}(5.9 \mathrm{~nm})$ photon energies at FERMI [8], which, along with the promising results from several other experimental and theoretical studies [15-19], is encouraging for its extension up to $620 \mathrm{eV}$ at LCLS-II.

Our studies on LCLS-II indicate that both EEHG and SXRSS can produce FEL pulses that are, at minimum, several times brighter than SASE and with narrower bandwidth spectra that are highly stable in central wavelength. SXRSS is the simplest and most mature seeding technology in the SXR regime; it also produces the highest photons/meV per pulse. EEHG has the advantages of excellent intensity stability for FTL pulses and the capability for tunable and phase-locked multi-pulse and multicolor operations, with the disadvantage that performance is projected to diminish significantly toward $1 \mathrm{keV}$ energies.

The EEHG and SXRSS options at LCLS-II are complementary and non-interfering. Components for EEHG can fit in the available $40 \mathrm{~m}$ space upstream of the SXR undulators in the current LCLS-II beam line design (see Fig. 1). They also can utilize some of the hardware intended for sub-fs pulse production [20,21] including magnetic undulators and chicanes. The SXRSS infrastructure (chicane magnets and SXR optics) can reside within a single undulator slot (as with LCLS).

There are unique and shared challenges with each scheme, including technical developments associated with the up to $1 \mathrm{MHz}$ rate LCLS-II system. For SXRSS, the primary issue is cooling of the $\mathrm{x}$-ray optics. For EEHG, this

TABLE I. Parameters of the LCLS-II e-beam for generating soft $\mathrm{X}$-rays.

\begin{tabular}{lccc}
\hline \hline Parameter & Symbol & Value & Unit \\
\hline Energy & $\mathrm{E}$ & 4.0 & $\mathrm{GeV}$ \\
Charge & $\mathrm{Q}$ & $50-300$ & $\mathrm{pC}$ \\
Peak Current & $\mathrm{I}$ & 1.0 & $\mathrm{kA}$ \\
Emittance & $\epsilon_{n}$ & 0.45 & $\mu \mathrm{m}$ \\
Energy spread & $\sigma_{E}$ & 500 & $\mathrm{keV}$ \\
Beta function & $\langle\beta\rangle$ & 12 & $\mathrm{~m}$ \\
\hline \hline
\end{tabular}

TABLE II. Parameters of the LCLS-II soft x-ray undulators.

\begin{tabular}{lcc}
\hline \hline Parameter & Value & Unit \\
\hline Type & Hybrid PM, planar & $\ldots$ \\
Full gap height & Variable & $\ldots$ \\
Period & 39 & $\mathrm{~mm}$ \\
Segment length & 3.4 & $\mathrm{~m}$ \\
Break length & 1 & $\mathrm{~m}$ \\
No. of segments & 21 & $\ldots$ \\
Total length & 96 & $\mathrm{~m}$ \\
\hline \hline
\end{tabular}


includes implementation of a suitable high-repetition rate, high power external UV laser system to manipulate the electron beam (e-beam), though seed laser operation at reduced rates is straightforward. Both seeding schemes also require excellent linear e-beam phase space properties for best operation, but in general may favor different parameters and bunch profiles for optimal performance.

\section{SEEDING PERFORMANCE COMPARISON}

Numerical seeding studies were performed with the FEL code Genesis [22] using two different e-beam distributions. One was a $100 \mathrm{pC}$ start-to-end (S2E) e-beam produced by the IMPACT [23] code that tracked electrons from the cathode source to the LCLS-II SXR undulator entrance. The other was a $50 \mathrm{pC}$ idealized version of the S2E e-beam with a lasing core of similar length (50 fs) but with a smooth longitudinal phase space distribution. Figure 2 shows the longitudinal phase space and current profiles of both e-beams [24]. The S2E e-beam was originally optimized primarily for low transverse emittance $\left(\epsilon_{n}=0.4 \mu \mathrm{m}\right)$ and high current $(\approx 1 \mathrm{kA})$, and is thus not necessarily optimized for seeding. Its phase space distribution shows clear energy structure that impacts the FEL performance, notably short IR wavelength ripples from microbunching, curvature in the e-beam core, a long tail, and strong nonlinearities at the head. Nevertheless, it serves as a useful benchmark to examine the seeding limitations caused by non-optimal e-beams. The ideal e-beam, which otherwise matches the properties of the S2E e-beam (emittance, slice energy spread, etc.), illustrates by contrast the impact of the phase space and current distortions on the FEL performance. We note that schemespecific, seed-optimized S2E e-beams with cleaner phase spaces are currently under active study. Figure 2 also shows the calculated resistive wall wakefields (RWW) of the SXR undulator vacuum chambers used in simulations.
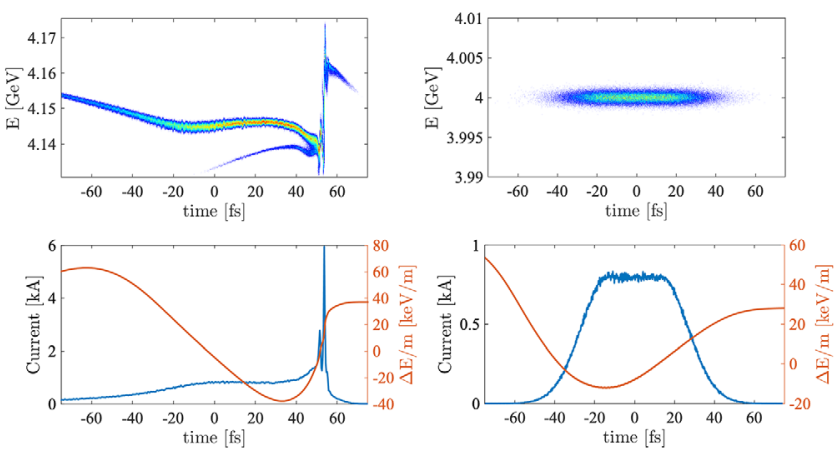

FIG. 2. Longitudinal phase spaces of the electron beams used in LCLS-II FEL simulations (head is to the right). The $100 \mathrm{pC}$ startto-end e-beam from IMPACT optimizations is on the left, and the $50 \mathrm{pC}$ ideal e-beam is on the right. Also shown: current profiles and time-dependent energy loss per meter in the undulator due to resistive wall wakefields (RWWs).

\begin{tabular}{|c|c|c|c|c|}
\hline \multirow[t]{2}{*}{ LCLS-II } & \multicolumn{2}{|c|}{ EEHG } & \multicolumn{2}{|c|}{ SXRSS } \\
\hline & $1 \mathrm{~nm}$ & $2 \mathrm{~nm}$ & $1 \mathrm{~nm}$ & $2 \mathrm{~nm}$ \\
\hline $\begin{array}{l}\text { Photons } / \mathrm{meV} / \text { pulse } \\
\text { (peak, 10 } 0^{\circ} \text { ) }\end{array}$ & $\begin{array}{c}1 \\
1.4\end{array}$ & $\begin{array}{c}4 \\
10\end{array}$ & $\begin{array}{l}38 \\
17\end{array}$ & $\begin{array}{l}180 \\
130\end{array}$ \\
\hline $\begin{array}{l}\text { Bandwidth }\left(\Delta E, \Delta E_{\mathrm{e}}\right) \\
{[\mathrm{meV}]}\end{array}$ & $\begin{array}{l}250,340 \\
125,350\end{array}$ & $\begin{array}{l}290,540 \\
180,200\end{array}$ & $\begin{array}{r}80,115 \\
130,205\end{array}$ & $\begin{array}{l}75,180 \\
90,140\end{array}$ \\
\hline \% of photons in FWHM & $\begin{array}{l}55 \% \\
60 \%\end{array}$ & $\begin{array}{l}60 \% \\
70 \%\end{array}$ & $\begin{array}{l}70 \% \\
60 \%\end{array}$ & $\begin{array}{l}55 \% \\
60 \%\end{array}$ \\
\hline$B_{e}\left(0.76 E / \Delta E_{e}\right)\left[10^{16}\right]$ & $\begin{array}{l}0.09 \\
0.07\end{array}$ & $\begin{array}{l}0.16 \\
0.58\end{array}$ & $\begin{array}{l}3.21 \\
0.36\end{array}$ & $\begin{array}{l}4.9 \\
2.6\end{array}$ \\
\hline Pulse Duration & \multicolumn{2}{|c|}{$1-25 \mathrm{fs}$} & \multicolumn{2}{|c|}{$1-25 \mathrm{fs}$} \\
\hline Spectral Stability & \multicolumn{2}{|c|}{ high } & \multicolumn{2}{|c|}{ high } \\
\hline Intensity Stability & \multicolumn{2}{|c|}{ high } & \multicolumn{2}{|c|}{ low } \\
\hline Complexity & \multicolumn{2}{|c|}{ high } & \multicolumn{2}{|c|}{ low } \\
\hline Two pulse/Multicolor? & \multicolumn{2}{|c|}{ yes/yes } & \multicolumn{2}{|c|}{ yes/no } \\
\hline
\end{tabular}

FIG. 3. Seeded LCLS-II performance comparison. $\Delta E_{e}$ is the FWHM equivalent of a Gaussian, and is the minimum spectral extent that contains $76 \%$ of the total energy, $E_{N}=N E_{p h}$. This incorporates spectral pedestal contributions that are missed by the conventional FWHM, $\Delta E$. All values calculated near saturation at the undulator position that gives the peak of $B_{e}=0.76 E_{N} /$ $\Delta E_{e}$, which is the number of photons contained within the relative FWHM equivalent bandwidth. For comparison, unmonochromatized SASE averages $10^{9}$ photons $/ \mathrm{meV}$ at $1.2 \mathrm{keV}$ and $4 \times 10^{9}$ photons $/ \mathrm{meV}$ at $620 \mathrm{eV}$. For comparison with other general features of monochromatized SASE see [2].

A summary of results from numerical simulations is shown for reference in Fig. 3, with the best output FEL spectra (obtained with the ideal e-beam) shown in Fig. 4. In general, both seeding schemes produce pulses with higher peak brightness than monochromatized SASE at the same saturation power, especially when the efficiency of any post undulator monochromator is included. The ideal e-beams produce cleaner x-ray spectra as expected, but the $50 \mathrm{fs}$ effective lasing core is roughly $10 \mathrm{fs}$ shorter than that of the S2E e-beam, which results in slightly larger bandwidths.

For the numerical results presented in Fig. 3, the spectral bandwidth of the FEL output is quantified in two different ways in order to capture the effect of the spectral pedestal (i.e., spectral content outside the main seeded spike), which can have a complicated shape. $\Delta E$ is the simple FWHM bandwidth of the most prominent spectral peak, while $\Delta E_{e}$ is the FWHM-equivalent bandwidth which is the minimum spectral extent that contains $76 \%$ of the total pulse energy $E_{N}=N E_{\mathrm{ph}}$, where $N$ is the number of photons. This is the bandwidth used to define the resolving power

$$
R=E_{\mathrm{ph}} / \Delta E_{e} .
$$

Also given in Fig. 3 is the percent fraction of the photons contained in the FWHM bandwidth. This can be compared to a range of ideal functions. For example, the percentage 

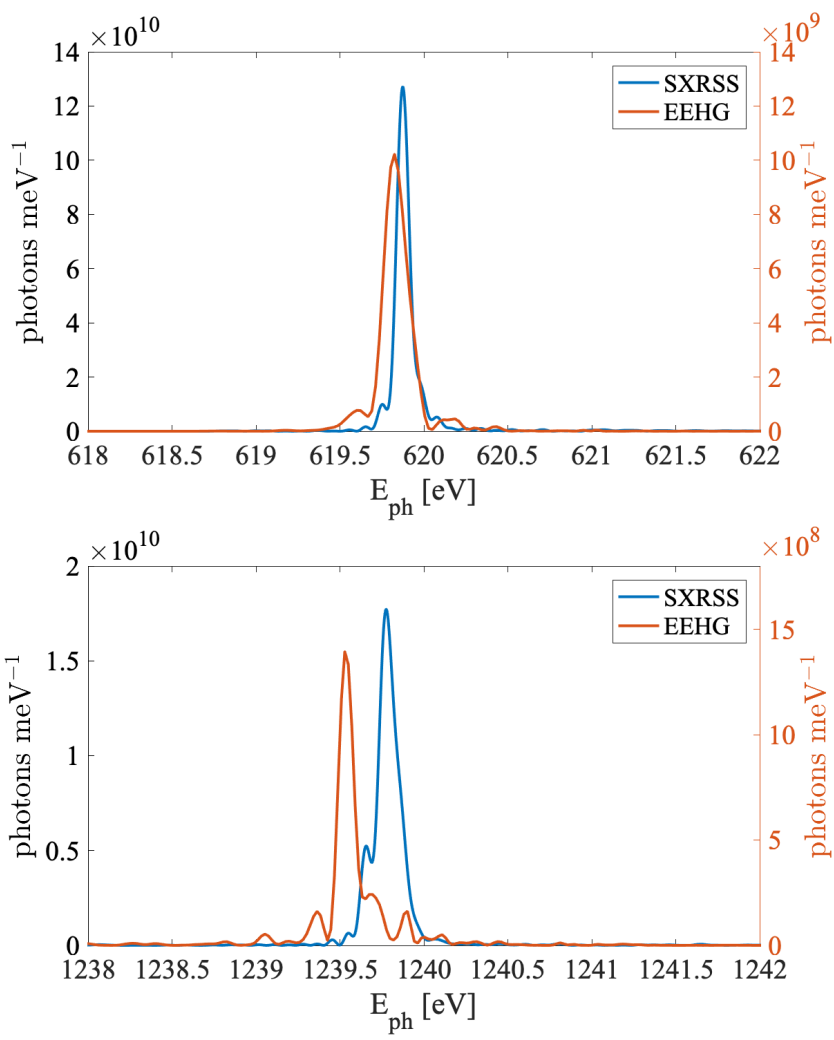

FIG. 4. X-ray spectra of SXRSS and EEHG near saturation for the ideal e-beam.

contained within the FWHM of a Gaussian distribution is $76 \%$, while a $\operatorname{sech}^{2}$ is $71 \%$, and a Lorentzian is $50 \%$.

The listed peak brightnesses and bandwidths are all calculated at the undulator position that gives the peak of the dimensionless brightness quantity $B_{e}=0.76 E_{N} / \Delta E_{e}=$ $0.76 N R$ which is the number of photons contained within the relative FWHM-equivalent bandwidth. The maximum of $B_{e}$ therefore algorithmically selects a location of peak dimensionless brightness that includes the FEL amplification and pedestal growth/spectral broadening, and thus $B_{e}$ is the "brightness" figure of merit used to quantitatively compare schemes. In most cases, this peak location tends to be very close to saturation, but it moves downstream toward the end of the undulator when tapering is included. Tapering and wakefields are both included in all simulations. Radiation temporal pulse durations all refer to the FWHM value unless otherwise stated. The "general features" listed in Fig. 3 are qualitative assessments informed by combinations of the present simulations and by the authors' varied experiences in previous numerical and experimental studies.

It is important to note that overall, while SXRSS and EEHG both improve the brightness of the FEL over monochromatized SASE, neither method uniformly achieves all of the desired characteristics of fully stable FTL pulses with a negligible spectral pedestal at or beyond saturation. In particular, the target bandwidth of $30 \mathrm{meV}$ for pulses with high spectral resolution is at least a factor of two below that achievable with the current LCLS-II design, due primarily to the RWW effects in the SXR undulator vacuum chambers (discussed later). Nevertheless, both schemes can produce pulses with specific desirable characteristics that can be targeted at distinct science applications. Details are described in the following sections.

\section{SXRSS}

Self-seeding relies on the monochromatization and reinjection of the FEL radiation pulse part way through the exponential gain regime to significantly increase the temporal coherence and spectral brightness over what is achievable with normal SASE [25]. It is the simplest scheme to implement, as it does not rely upon external lasers with their issues of repetition rate capability and synchronization. SXRSS has been a core capability at LCLS [3,26,27], and the physical infrastructure will be moved to the LCLS-II SXR line, upgraded for cooling and motion control.

SXRSS experiments at LCLS demonstrated an increase in peak brightness by a factor of up to 20-50 over monochromatized SASE (2-5 if the seeded output is also monochromatized) across the photon energy tuning range of $0.5-1 \mathrm{keV}$ [3], though the improvements over SASE can depend sensitively on the details of the setup and electron beam, as well as the requirements of the application $[4,28]$. The measured resolving power is roughly $R=2000-5000$, determined both by the monochromator grating and focusing optics for the seed x-rays, with the e-beam acting as its own exit slit. The relative variation in wavelength is stable to within $2 \times 10^{-5}$ for on-energy e-beams [28].

The measured LCLS SXRSS spectrum typically has a large seeded spike surrounded by a broad spectral pedestal. An extensive set of experimental studies on understanding and controlling the spectral contamination outside the main seeded line are reported in [28]. Studies for LCLS and LCLS-II indicate that there are three primary sources of spectral broadening during lasing, each with different spectral characteristics: (i) Microbunching instability (MBI). Historically the dominant source of the spectral pedestal, the MBI produces IR wavelength $\sim 0.3-5$ micron $(\sim 0.24-4 \mathrm{eV})$ structures in the e-beam phase space that frequency-mix with the seed to produce a shot-to-shot varying spectral pedestal. The pedestal grows at least linearly relative to the main seeded line prior to saturation, unlike the SASE component (see below). The total integrated pedestal can be controlled in part by the laser heater; down to $10 \%$ of the seed energy at LCLS [28]. (ii) Wakefields. The resistive wall wakefield (RWW) in the 5-mm aperture undulator vacuum chamber introduces a long-wavelength nonlinear chirp (see Fig. 2) that broadens the seeded x-ray spectrum prior to saturation. For the $1 \mathrm{kA}$, $4 \mathrm{GeV}$ e-beam at LCLS-II, this limits the maximum linear portion of the e-beam to $\sim 25 \mathrm{fs}(R \sim 10000$ at $1 \mathrm{keV})$. 
An increase in the aperture size from 5-mm to 7-mm or larger would virtually eliminate this contribution [29]. (iii) SASE and saturation. A weak or nonuniform x-ray seed along the e-beam raises the relative SASE background. Further, limitations on the allowable $20 \mathrm{~mJ} / \mathrm{cm}^{2}$ fluence on the SXRSS monochromator optics directly limits the ratio of seed to SASE power in the second stage [30]. At LCLS, SASE usually accounts for $\sim 10 \%$ of the total output pulse energy in the linear gain regime. Deep in saturation and in the absence of optimized tapering, the relative SASE contribution continues to increase, which drives up the pedestal.

Spectral broadening aside, the SXRSS pulses are not single spike nor transform-limited unless the electron bunch length matches the coherent pulse length set by the SXRSS system. For example, at LCLS with $R=5000$ for $1 \mathrm{keV}$ photons (i.e., $200 \mathrm{meV}$ FWHM), the transformlimited pulse duration is $\tau=9 \mathrm{fs}$. Thus, the standard $T=70$ fs flattop e-beam will be statistically seeded by $M=2.35 T / 2 \sqrt{\pi} \tau \approx 5$ individual temporal modes. From the standpoint of fluctuations, this stabilizes the energy within the seeded bandwidth (normalized fluctuation level $\sqrt{1 / M}$ ) [28], but leads to less strongly seeded regions of the e-beam where SASE dominates, which adversely impacts the pedestal [31]. Conversely, a 14 fs e-beam would produce single spike $(M \approx 1)$ nearly FTL $\mathrm{x}$-ray output, but with $\sim 100 \%$ pulse-to-pulse intensity fluctuations. Deep in saturation the intensity stabilizes, but the $\mathrm{x}$-ray spectrum can degrade due to sidebandlike growth [3].

\section{A. LCLS-II SXRSS Simulations}

The SXRSS photon seed pulse is modeled to be a $40 \mathrm{~kW}$ $(\approx 50$ times the shot noise) ideal pulse, fully covering the e-beam in time to establish best performance. The spectra are thus 2-3 times narrower than those expected from the migrated baseline LCLS SXRSS system with $R \approx 5000$;

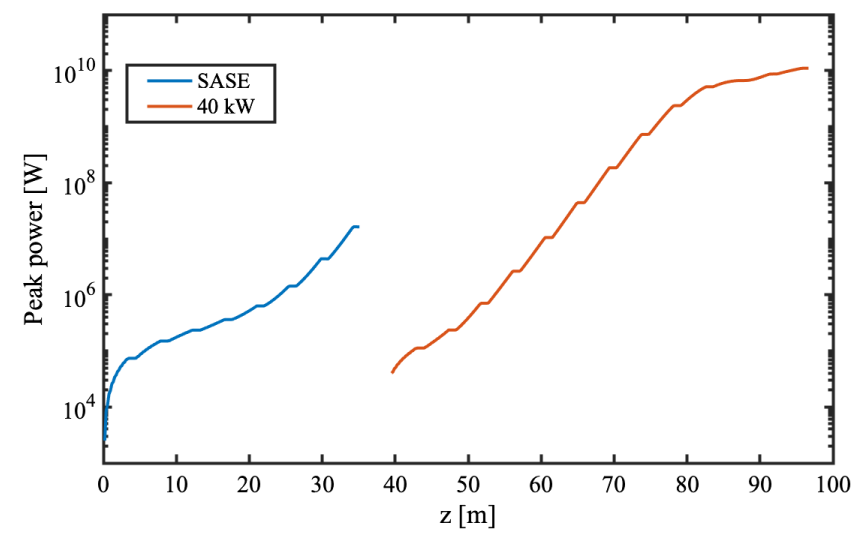

FIG. 5. Power evolution of $1 \mathrm{keV}$ x-rays during SXRSS at LCLS-II. Here and throughout this paper the SXRSS infrastructure is modeled in the slot 34 location.

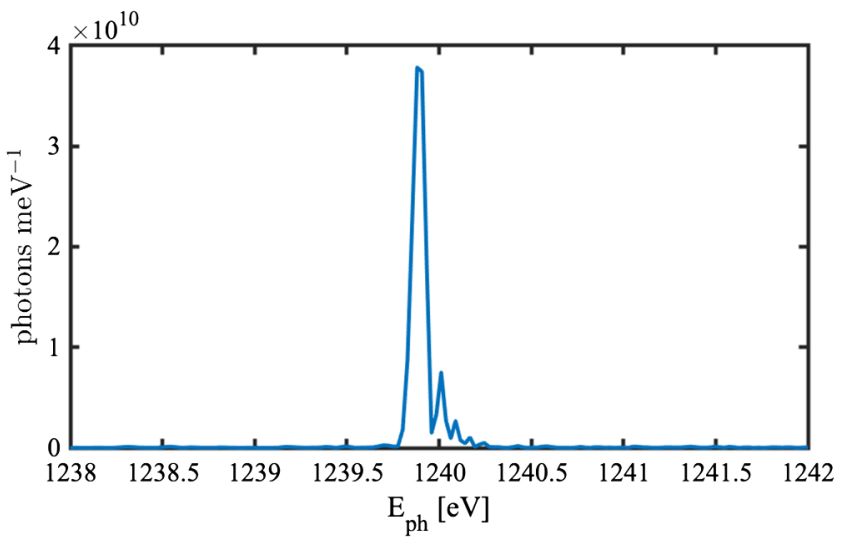

FIG. 6. SXRSS with S2E e-beam. $1 \mathrm{keV}$ spectrum at the end of the undulator. The spectral behavior at $620 \mathrm{eV}$ (not shown) is very similar.

however these narrow bandwidths may be achievable with upgraded monochromator optics.

Figure 5 shows the power growth of a $1 \mathrm{keV}$ FEL pulse from the S2E e-beam along the LCLS-II SXR line, including the initial SASE portion. The final amplified
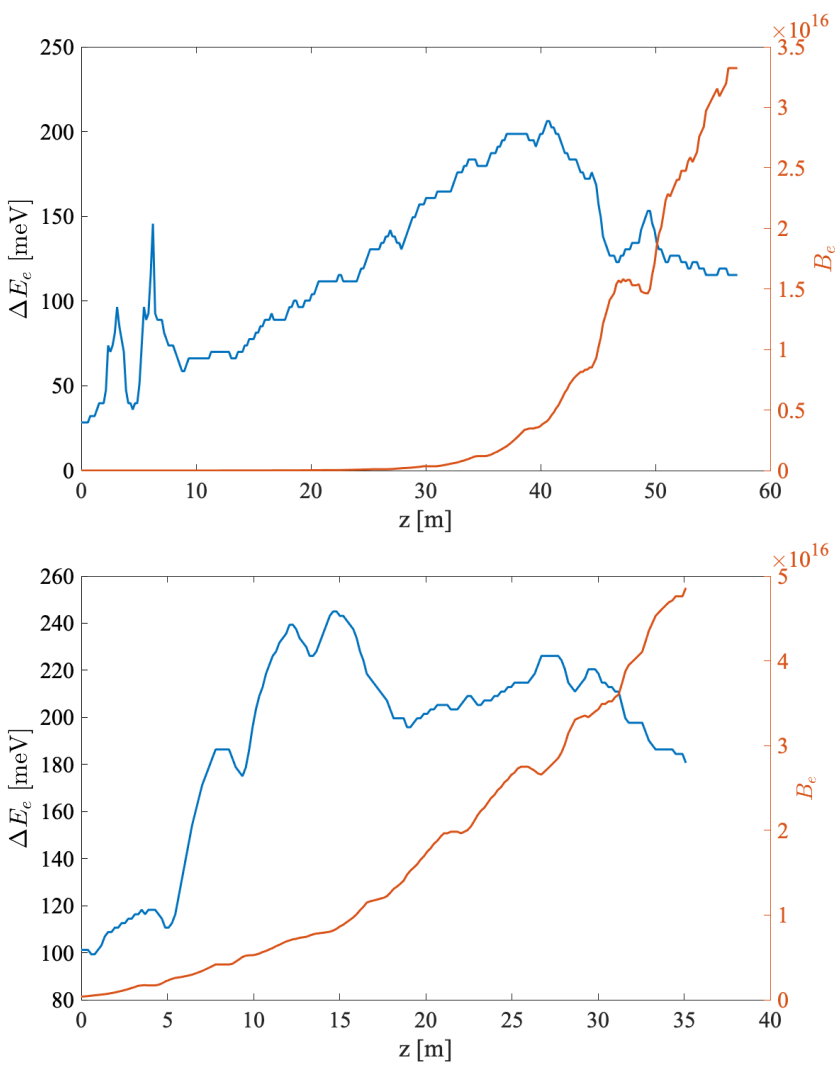

FIG. 7. SXRSS x-ray spectral bandwidth and brightness evolution with S2E e-beam at $1.2 \mathrm{keV}$ (top) and $620 \mathrm{eV}$ (bottom). The FWHM-equivalent bandwidth $\Delta E_{e}$ first grows then is reduced somewhat near saturation as the taper begins. 

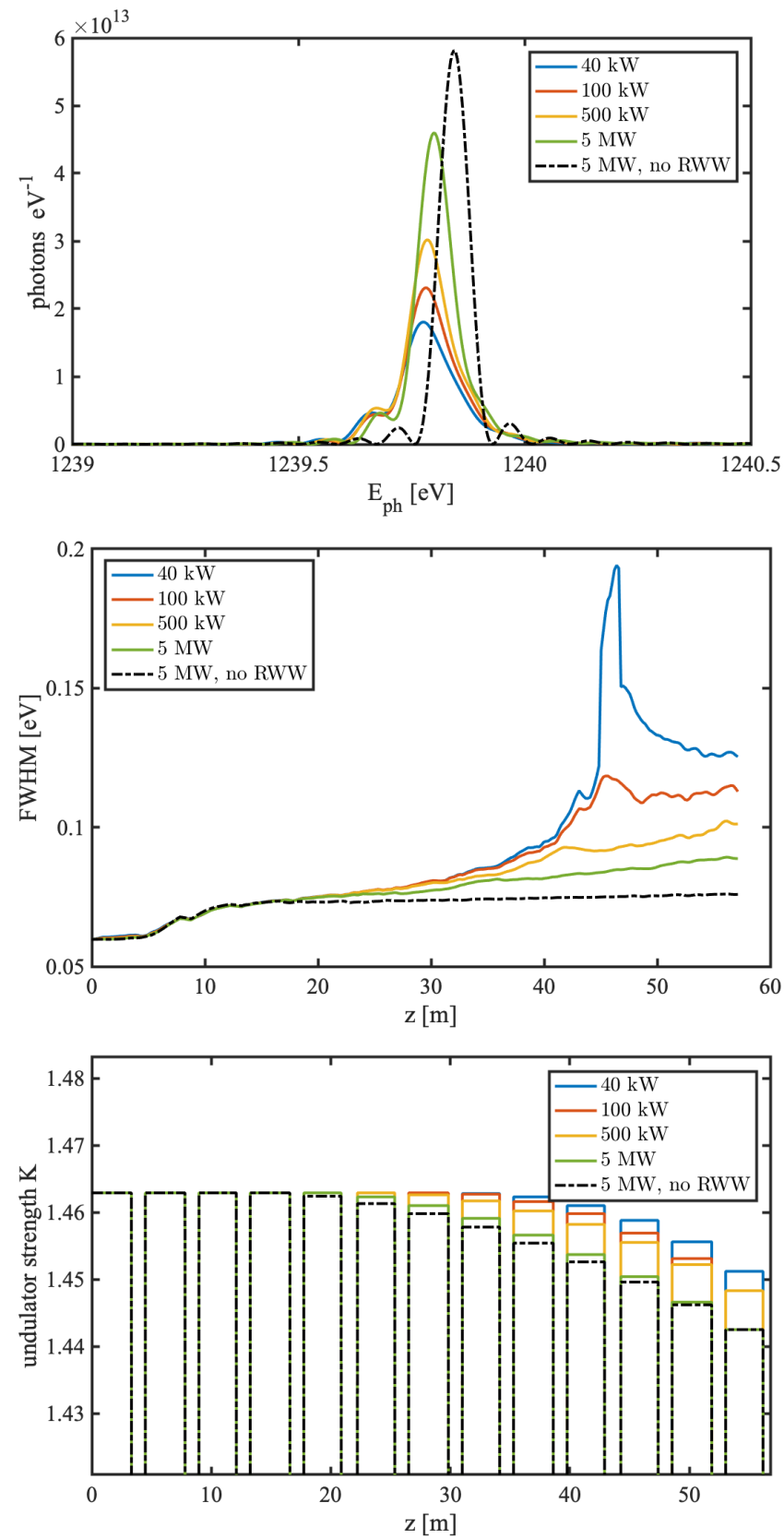

FIG. 8. SXRSS with ideal 50 fs, $1 \mathrm{kA}$ flattop e-beam for different input powers on ideal seeds. Top: final spectra, Middle: bandwidth evolution, Bottom: optimized taper profiles.

seed pulse reaches the $10 \mathrm{GW}$ level, and the output spectrum is shown in Fig. 6. The final spectral bandwidth is 2.5 times the FTL. It has a narrow $\Delta E=80 \mathrm{meV}$ spike and smaller nearby pedestal structure driven primarily by MBI modulations and some RWW-induced nonlinear chirp during lasing. The growth of the spectral bandwidth is shown as a function of distance from the SXRSS system in Fig. 7. The bandwidth grows steadily as the pulse approaches saturation $(40 \mathrm{~m}$ downstream in the $1.2 \mathrm{keV}$ case, $15 \mathrm{~m}$ downstream for $620 \mathrm{eV}$ ), but the tapered sections of undulators serve to mitigate the growth while extracting more pulse energy.

For comparison and to establish baseline expectations, simulations were performed with the ideal e-beam. It has no initial MBI or nonlinear phase space structure, but the RWW in the undulators is still included. An ideal radiation seed is again assumed. Figure 4 shows the essentially single spike output spectra (both $\approx 1.5$ times the FTL).

For LCLS-II, the RWW is the main limiting factor for obtaining resolving powers beyond $R=10000$ $\left(\Delta E_{e}=100 \mathrm{meV}\right)$. The RWW impact on the ideal e-beam is indicated in Fig. 8 where the seed bandwidth $\Delta E$ along the undulator is plotted for different seed powers, ranging from the nominal $40 \mathrm{~kW}$ level up to $5 \mathrm{MW}$ (corresponding to a saturated LCLS-II e-beam monochromatized). These simulated seed power scans were performed to reveal if the RWW-induced broadening could be outrun, thereby motivating a redesign and/or major reposition of the LCLS-II SXRSS chicane to accommodate high power. Scans show that this solution may be impractical; the peak photons/eV improves by a modest 2.5 times for a significant seed increase from $40 \mathrm{~kW}$ to $5 \mathrm{MW}$, while the time-bandwidth product improves from 1.5 to 1.16 . This suggests that achieving bandwidths much less than $100 \mathrm{meV}$ would require significant effort; either larger vacuum chamber apertures (e.g., increase from $5 \mathrm{~mm}$ to $7 \mathrm{~mm}$ ) to remove RWW effects, or a factor of 100 increase in the seed power.

\section{EEHG}

The echo-enabled harmonic generation (EEHG) scheme uses two external $260 \mathrm{~nm}$ lasers to produce a high harmonic coherent density modulation (bunching) in the e-beam to seed the SXR FEL $[13,14]$. The bunching is produced by a series of transformations on the electron phase space distribution. The first laser imprints a sinusoidal energy modulation (2-3 times the slice energy spread $\sigma_{E}$ ) in the e-beam as they copropagate through a magnetic undulator (modulator). The phase space distribution is then highly dispersed as the e-beam goes through a strong chicane (nominal $R_{56(1)} \sim 15 \mathrm{~mm}$ ) which filaments the phase space into energy bands. The second laser pulse then energy modulates the e-beam again (by $4-6 \sigma_{E}$ ). After passing through the second weaker chicane $\left(R_{56(2)} \sim 0.1 \mathrm{~mm}\right)$, bunching at the desired SXR wavelength is produced. The bunched e-beam then enters the FEL where it radiates and amplifies coherent light up to saturation levels. The central bunching frequency is a sum of harmonics of the two laser frequencies, $\omega_{E}=h \omega_{1}=n \omega_{1}+m \omega_{2}$ so it can be adjusted by changing one or both of the laser frequencies. Optimal bunching requires $n=-1$. Thus $m \approx h \gg 1$, and the bunching spectrum is much more sensitive to the second laser [32,33]. Maximizing the bunching to occur at a specific harmonic is chosen by proper choice of the chicane dispersions, $h \approx|n| R_{56(1)} / R_{56(2)}$. 


\section{A. EEHG characteristics}

By the use of external lasers, EEHG offers additional control on the output FEL pulse characteristics because the seed lasers can be tailored in pulse duration, amplitude, and wavelength. The spectral phase of the seed lasers (especially the second laser) can also be specified (by the introduction of a chirp, for example) to control or even correct for the spectral phase of the output FEL pulse (e.g., [34]). Here we concentrate on producing the longest pulses with good output spectra. However, short pulses down to a few fs are possible in principle by using a short second laser (and shorter modulator) if SASE in the rest of the e-beam can be appropriately limited [35].

Nominal expected FEL pulse lengths at LCLS-II with EEHG are $1-20$ fs with $\Delta \omega / \omega_{E} \sim 1-20 \times 10^{-4}$ FWHM bandwidths (depending on the wavelength), defined primarily by the second laser pulse duration $\sigma_{L} / c$ but limited to $<25-30$ fs by wakefields. FEL spectra and temporal pulse profiles from ideal e-beam simulations are shown in Fig. 9. With tapering, the EEHG pulses reach the $10 \mathrm{GW}$
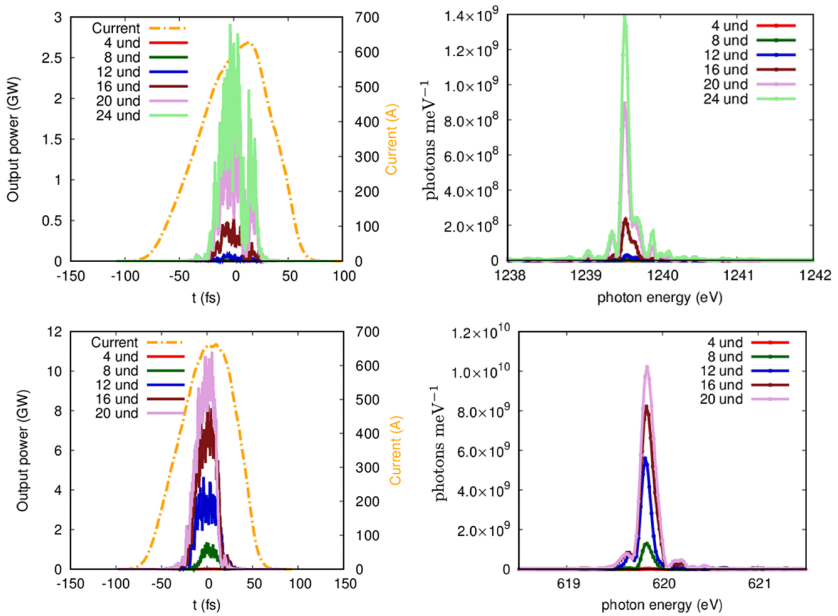

FIG. 9. EEHG with ideal e-beam at $1.2 \mathrm{keV}$ (top) and $620 \mathrm{eV}$ (bottom). Power profiles overlaid with the final current profile (left) and spectra (right) at different positions along the radiating undulators. Note that, for clarity, the current profiles are averaged over the wavelength of the UV laser seeds.
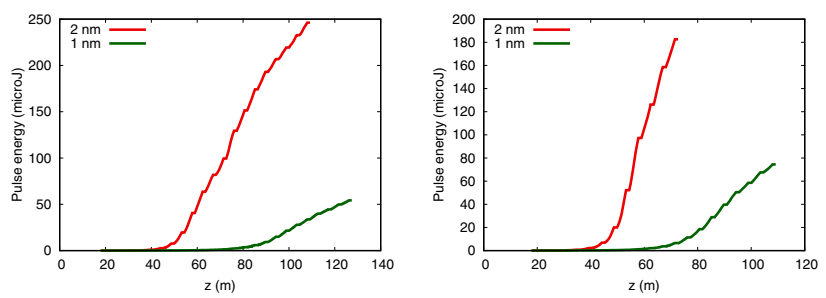

FIG. 10. EEHG gain curves with the ideal beam (left) and the $\mathrm{S} 2 \mathrm{E}$ beam (right). level (see Fig. 10), which is comparable to that achieved by SXRSS.

In these simulations and in general, it is optimal to keep the first laser pulse at least as long as the second laser pulse to serve as a buffer against timing jitter and to suppress SASE. The minimum bandwidth is obtained near the optimum second laser modulation, $\Delta E_{2} / E=\lambda_{2} / 2 \pi R_{56(2)}$. At this value the bunching envelope is shorter than the laser pulse by the factor $h^{1 / 3} / 1.4$ due to a harmonic compression effect $[5,32,33]$. Thus, to produce a $20 \mathrm{fs}$ bunching envelope at $h=130$, one needs a $70 \mathrm{fs}$ laser pulse. Variation in the e-beam current profile, however, can also impact the FEL pulse length due to preferential lasing.

For laser pulses short compared to the electron bunch duration, the time-integrated bunching is relatively robust to laser power fluctuations, resulting in an FEL intensity that can be even more stable than SXRSS [8]. This is illustrated in Fig. 11. For example, 5\% variation from the optimum second seed laser power corresponds to $10 \%$ integrated bunching fluctuations at $h=130$ (20\% at $h=260$ ). The bunching bandwidth, which also fluctuates with the laser power, shows a comparable $5 \%$ fluctuation at $h=130(10 \%$ at $h=260)$.

The growth of the bunching bandwidth for larger than optimal laser power identifies the onset of spectral pulse splitting, which can be utilized for multicolor operations. In fact, EEHG has the general capability to produce phaselocked double pulses with adjustable timing delay, or multiple colors using techniques pioneered with HGHG [9-11,36]. For example, an over-modulation with the second laser as shown in the top plot of Fig. 11 can eventually split the harmonic as a result of temporally separated pulses that interfere. If the laser is also chirped, two distinct colors with adjustable delay are produced [36]. Or, two seed pulses can modulate the e-beam in the second modulator, much like that demonstrated in Ref. [11] with split and delay. This offers some timing control between pulses, and also offers the possibility of different FEL colors if two seed lasers with different wavelengths are used. Another possibility is to harness the energy chirps in the e-beam produced by wakefields to split the spectrum. Simulations of these two color operations are described below. Alternately, significant bunching at multiple nearby harmonics is parasitically produced with widely spaced $\left(\Delta \omega / \omega_{E}=1 / h\right)$ color separation [8]. This option requires a split FEL undulator configuration (e.g., consecutive or interleaved) since the $1 / h$ separation is typically larger than the FEL bandwidth. This comes at the expense of output power because neither color reaches saturation. Nevertheless, multiple pulses with lower FEL output energies still have several desirable applications such as time-resolved resonant processes. If relative phase stability between colors is also achieved, then one could foresee an extension of molecular vibrational wavepacket interferometry to electronic wavepacket interferometry $[37,38]$. 

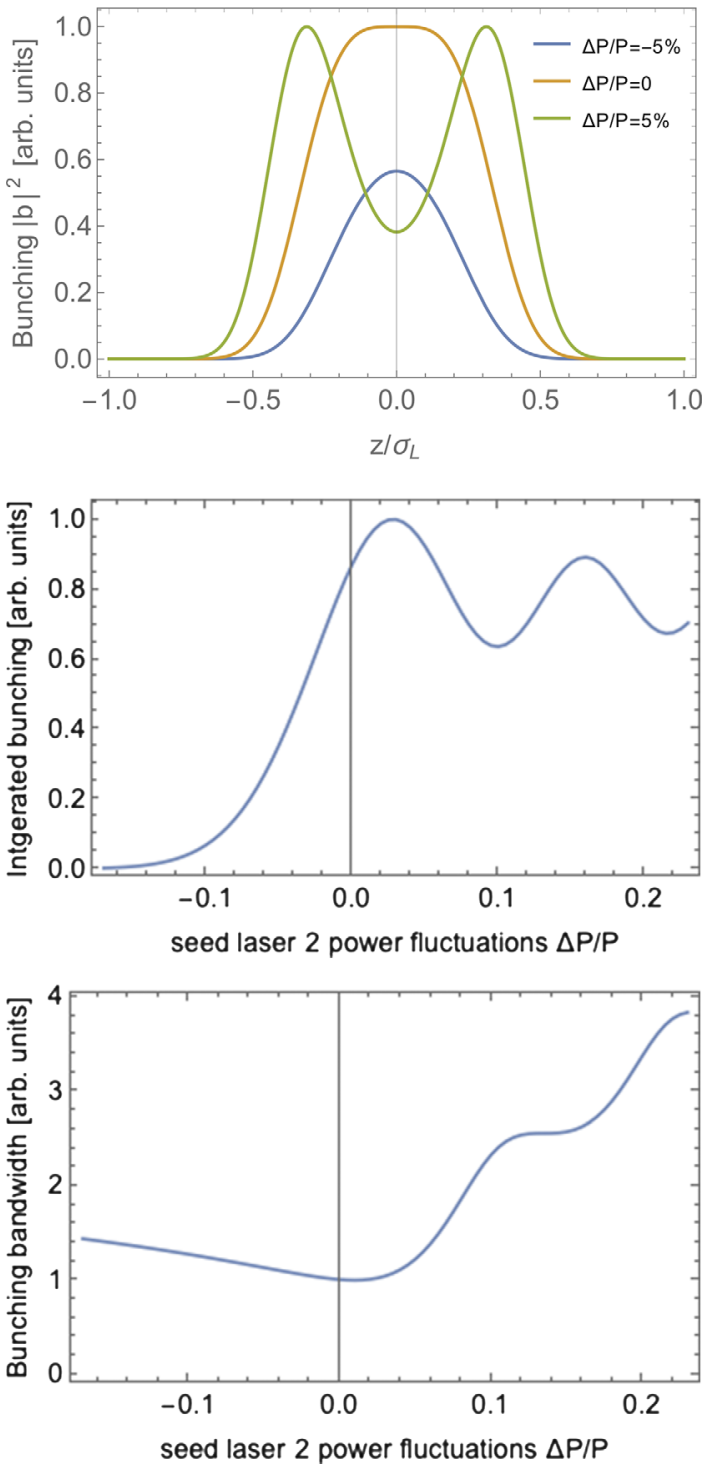

FIG. 11. Effects of intensity fluctuations in the second EEHG laser from theory for $h=130$. The temporal bunching profile becomes split by over-modulation (top), which enables multicolor seeding through interference between the two phase-locked pulses. The integrated bunching power (middle), and the bunching bandwidth (bottom) vary accordingly.

\section{B. EEHG challenges}

Studies point to several critical issues for optimal performance, most of which relate to aspects of the laser system or beamline insertion devices $[16,39]$. These effects are all included in the simulations presented here, and the mitigating solutions discussed are implemented to achieve the best FEL performance (i) Laser spectral phase: In the simplest models, eikonal phase structure in the second laser gets multiplied by the large harmonic factor $m[32,33,40,41]$. However, this detrimental effect is suppressed by the combination of harmonic compression and slippage in the modulator $[42,43]$. Slippage smooths out laser phase errors if it is comparable in duration to the final $<30 \mathrm{fs}$ bunching

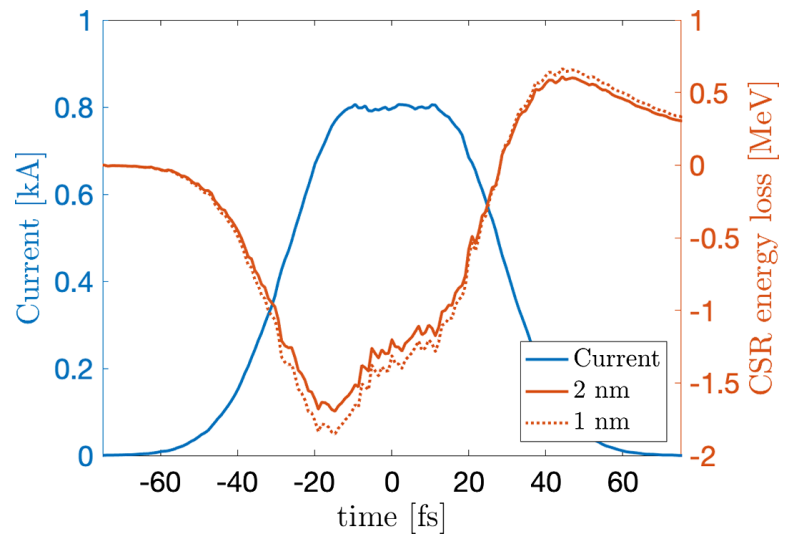

FIG. 12. Steady state CSR energy loss in the ideal beam in the last two chicane dipoles, where the beam is highly sensitive to energy distortions. The wake profiles are similar between the 1 and $2 \mathrm{~nm}$ EEHG tunes despite the difference in dispersion because of the weak dependence of the CSR on the different magnetic fields.

envelope, which is 35 undulator periods with $260 \mathrm{~nm}$ lasers [44]. Phase wander in the bunched region may be required to be of order a couple degrees or less. For example, dominant quadratic temporal phase variations over the envelope should be $<1 / 2 h^{1 / 3}$ radians to keep the bunching bandwidth within a factor of 2 of the FTL minimum [45]. In addition, deterministic laser phase shaping can be ameliorative, particularly if used to compensate e-beam energy chirp [34]. (ii) MBI: The strong EEHG modulators required to couple $260 \mathrm{~nm}$ lasers with the $4 \mathrm{GeV}$ e-beam can increase MBI effects $[45,46]$, which are particularly detrimental as they cascade into the second modulator. This motivates small $K$ modulators, and strong suppression of initial MBI by the laser heater. The rms energy spread growth through the second modulator should ideally be less than $\sigma_{\mathrm{MBI}}<$ $h^{1 / 3} \sigma_{E} / 3 k_{2} \sigma_{L}$ for modulations that produce sidebands within the FEL bandwidth. (iii) Incoherent synchrotron radiation (ISR): The transit of the e-beam through the modulators and chicanes induces ISR energy spread growth that can reduce the bunching, particularly if it occurs in the strong chicane and second undulator. Again, the second modulator $K$ cannot be too strong nor the chicane bends too short. For a fixed $R_{56}$ and total chicane length $L=2 L_{D}+$ $4 L_{m}$, the optimal magnet length is $L_{m}=3 L / 14$ to minimize ISR $\left(L_{D}\right.$ is the drift length between dipoles $1 \& 2$ and $\left.3 \& 4\right)$. (iv) Coherent synchrotron radiation (CSR): CSR wakes in the strong chicane produce long wavelength energy structures in the e-beam (see Fig. 12) that broaden the bunching spectrum [45]. Reducing CSR generally favors gentle bends, flatter current profiles, and short second laser pulses that select only the most linear regions of the wake. The impact of CSR is also sensitive to the peak current. Studies indicate that the CSR from the final two dipoles in the first chicane has the greatest impact on the spectrum. (v) Intrabeam scattering (IBS): IBS effects increase with 
beam density and with transport length [47-50], favoring a compact EEHG layout, in conflict with ISR requirements for large, weak magnets. IBS increases the energy spread and reduces the bunching. These effects are most critical at the locations where the beam phase space is sheared. Our studies with the proposed LCLS-II EEHG layout indicate $~ 15 \%$ total bunching degradation from IBS and ISR at $1.2 \mathrm{keV}$. (vi) Large amplitude e-beam energy structures: Passage through the large chicane can substantially change the e-beam phase space and current profile, particularly if there are large energy chirps in the head or tail. The large negatively chirped energy tail in the examined S2E beams, for example, becomes compressed and can even overlap the core and spoil the final FEL spectrum [51].

\section{LCLS-II EEHG simulations}

EEHG simulations were performed with Genesis using the LCLS-II S2E and ideal beam distributions. Parameters are shown in Table III. The compact system under study occupies a $20 \mathrm{~m}$ section (the equivalent of 5 undulator slots) upstream of the SXR undulator (Fig. 1). The modulators have strengths $K_{1}=25$, and $K_{2}=12.5$. The second modulator has a longer period and is weaker in strength to avoid deleterious energy scattering and MBI effects on the e-beam phase space. Each of the four-dipole symmetric chicanes use magnets with identical fields and length, as well as drifts of identical length. The 260-nm, GW-class seed lasers are focused to a 300 micron waist in the middle of the modulators (equivalent to $1 \mathrm{~m}$ Rayleigh lengths). As was true for the SXRSS simulations, undulator tapering (quadratic in undulator strength) is used to optimize the spectral performance.

Simulations indicate that the ideal beam can produce an output spectrum that is within $3 x$ the FTL. For the example at $1.2 \mathrm{keV}$ shown in Fig. 9, the output radiation pulse has a FWHM of $30 \mathrm{fs}$ and spectral FWHM of $125 \mathrm{meV}$. In this case, long $400 \mathrm{fs}$ seed lasers were used, as the sharpness of the current profile sets the length of the lasing core. The final brightness $B_{e}$ is about $5 \mathrm{x}$ less than was produced by SXRSS with the ideal beam, and there is a significant SASE pedestal due to the low $1 \%$ seeded bunching factor. At $620 \mathrm{eV}$ the situation improves, producing a pedestal less than 10\% (less than half that of SXRSS), and a brightness that is $4 \mathrm{x}$ less than SXRSS. The $n=-1$ EEHG tune is used in both cases.

TABLE III. EEHG parameters used in LCLS-II simulations.

\begin{tabular}{lrcc}
\hline \hline Element & Strength 1(2) nm & Magnet Length & Total Length \\
\hline Mod 1 & $\Delta E_{1}=1.5(1) \mathrm{MeV}$ & $\lambda_{u}=10 \mathrm{~cm}$ & $3.2 \mathrm{~m}$ \\
Mod 2 & $\Delta E_{2}=3(2) \mathrm{MeV}$ & $\lambda_{u}=40 \mathrm{~cm}$ & $3.2 \mathrm{~m}$ \\
Chic 1 & $R_{56(1)}=14(9.8) \mathrm{mm}$ & $L_{m}=2 \mathrm{~m}$ & $9.25 \mathrm{~m}$ \\
Chic 2 & $R_{56(2)}=53(85) \mu \mathrm{m}$ & $L_{m}=25 \mathrm{~cm}$ & $2.25 \mathrm{~m}$ \\
\hline \hline
\end{tabular}

The large chromatic dispersion of the first EEHG chicane transforms the initially flat-top 50 fs current profile into a nearly triangular distribution with a reduced peak current, resulting in a narrowed central laser core. This sets the maximum effective output pulse length to $30 \mathrm{fs}$, and also reduces the number of electrons that participate fully in the FEL amplification, so the total output power is reduced. The linear region of the CSR wake (Fig. 12) is also shorter for short beams, so we find that longer linear beams can perform slightly better with EEHG. For example, simulations with 100 fs flattop beams show peak brightness of $B_{e}=1 \times 10^{15}$ without tapering, compared to $B_{e}=0.7 \times$ $10^{15}$ with $50 \mathrm{fs}$ beams and tapering. In the absence of CSR, the $1.2 \mathrm{keV}$ spectrum is significantly cleaner [51].

EEHG simulations using the S2E beam indicate an overall poor spectral performance due primarily to the large energy structures on the beam. These lead to strong nonlinear distortion of the longitudinal phase space in the large chicane that produce strong current spikes which also drive sharp CSR wakes, and ultimately limit the lasing core to a short temporal region. As shown in Fig. 13, optimization efforts at $1.2 \mathrm{keV}$ show it is possible with $400 \mathrm{fs}$ lasers to produce a spectral spike that is $250 \mathrm{meV}$ FWHM ( $3 \mathrm{x}$ the transform limit) and contains $2.8 \times 10^{11}$ photons; a factor of 35 smaller spectral brightness than was produced by SXRSS simulations. At $620 \mathrm{eV}$, the EEHG spectrum is not much better due to interference effects between different temporal lasing portions.

One potential remedy for the large energy structure problem is to apply an energy cut to the e-beam upstream of the undulators, if technologically possible at the desired repetition rate. Simulations of S2E beams cut within a $\pm 5 \mathrm{MeV}$ window indicated a somewhat improved spectral quality, but the SXR pulse is still short and there is a significant spectral pedestal. In this case, it was more
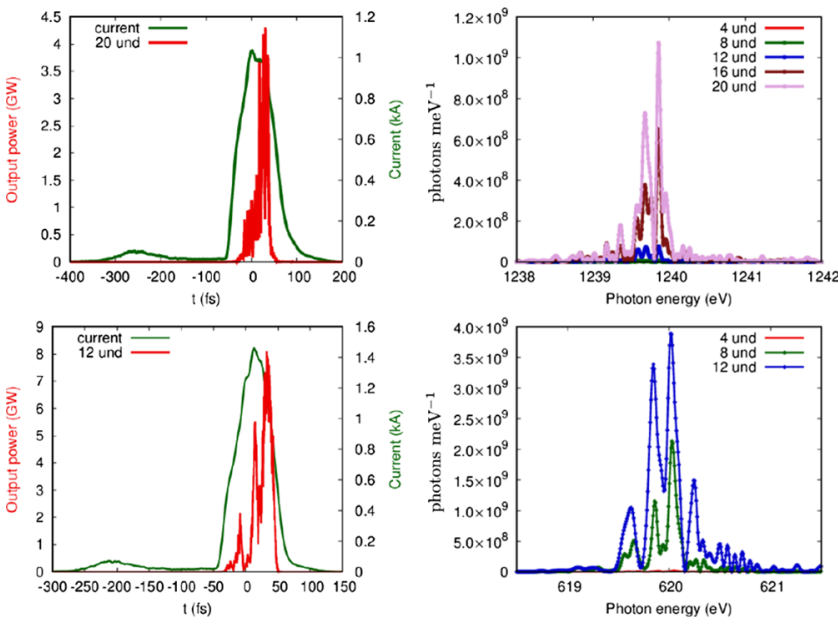

FIG. 13. EEHG x-rays at $1.2 \mathrm{keV}$ (top) and $620 \mathrm{eV}$ (bottom) with S2E e-beam. Power profiles overlaid with the final current profile (left) and spectra (right) at different positions along the radiating undulators. 
optimal to use seed lasers with a duration of 100 fs. These results highlight the importance of high quality e-beams for EEHG seeding.

In general, moderate initial modulations (e.g., MBI) mostly impact the EEHG scheme through wakefields, especially CSR in the first chicane and longitudinal space charge in the second modulator. In the extreme case, energy modulations may generate significant current spikes or folding over of the longitudinal beam phase space. Examples where an artificial energy modulation equal to that of the nominal $500 \mathrm{keV}$ uncorrelated (slice) energy spread has been applied to the idealized electron bunch have been studied. At $1 \mathrm{keV}$, The final pulse energy is reduced by $50 \%$ on average, due mostly to the reduction in bunching.

\section{Multicolor/Multipulse Operations}

One of the simplest ways to produce multicolor FEL pulses is through interference of multiple pulses to generate fine structures in the spectrum. This approach leverages the correlated nature of the radiation phase between temporally separated pulses. Two particular examples are shown in Fig. 14. The first is produced by increasing the power of the second laser seed to produce several local optima in the bunching (with phases of different sign) at different locations in the electron bunch. As a result of interference, there are multiple spectral peaks each separated by roughly $110 \mathrm{meV}$. The radiation is shown after 8 undulator sections tuned to the same $620 \mathrm{eV}$ energy. The spectrum with this technique is straightforward to adjust between single and multiple colors by the laser amplitude.

The second example of multicolor operation applies to moderate length $(100 \mathrm{pC})$ bunches seeded by long laser pulses, where wakefields can split the spectrum into multiple
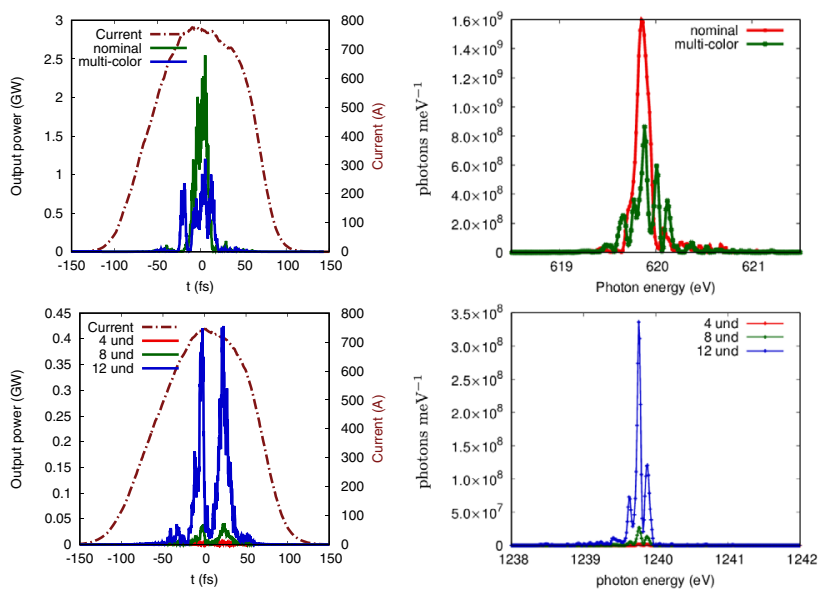

FIG. 14. Multicolor pulses with EEHG from an idealized $100 \mathrm{pC}$ bunch. Top: Radiation at $2 \mathrm{~nm}$. The second seed laser is increased slightly in power to produce the multi-color radiation. Bottom: Radiation at $1 \mathrm{~nm}$. Here CSR wakefields result in distinct spikes in the temporal profile and in the spectrum. components. CSR wakes from the first chicane are sufficiently intense and vary rapidly enough that the magnitude of the bunching is itself modulated, as shown in the lower left plot of Fig. 14. Here, there is no bunching and thus no radiation in the center of the beam, even though it is lasermodulated. The peaks in the resulting spectrum are separated by $130 \mathrm{meV}$. In this configuration the multicolor spectrum can be transformed to a single spike by using a shorter laser pulse for the second seed. For X-ray energies lower than $1.2 \mathrm{keV}$, the magnetic fields in the chicane can be reduced at the expense of increasing the power in the second laser. This would further reduce CSR wakefields, possibly to the point of avoiding large variations in bunching and allowing phase variations alone to yield either multiple colors or a frequency chirp within a single, long temporal pulse.

\section{CONCLUSIONS}

High-resolution numerical simulations indicate that EEHG and SXRSS are both promising but challenging options to produce high brightness SXRs at LCLS-II. For both schemes, flat and linear phase space distributions with minimal energy tails lead to better performance, and RWW effects limit the maximum FTL pulse to 25-30 fs unless the undulator vacuum chamber aperture is increased.

SXRSS at LCLS-II is predicted to deliver FEL pulses with $B_{e} \sim 5-35$ times higher spectral brightness than those from EEHG, depending on the e-beam characteristics, and has a greater advantage at shorter wavelengths. With a fixed resolving power grating (as in the LCLS design), the FTL pulse length is also fixed at a given photon wavelength (e.g., 9 fs at $1.2 \mathrm{keV}$ assuming $R=5000$ ). Thus, the e-beam must be either similarly short, or be properly sliced or spoiled (e.g., via laser heater shaping [52] or a "fresh slice" configuration as would be produced by a dechirper [53]) for FTL pulses. Such single mode operations translate to large output intensity fluctuations because the seed is a single mode starting from noise, although this is mitigated to some extent by saturation effects and undulator tapering.

EEHG has a potential to provide 1-25 fs ouput pulses within a few times the FTL, as well as temporally-tailored SXR pulses (e.g., multiple pulses/colors) at reduced brightness. Several effects constrain the maximum harmonic jump, including CSR and MBI effects in the EEHG transport. As such, producing high-quality pulses at full saturation power up to $1.2 \mathrm{keV}$ photon energies starting from UV seed wavelengths is beyond the reach of the current LCLS-II design. However, EEHG performance and flexibility improves at energies $620 \mathrm{eV}$ and lower. Stable, highquality UV lasers are critical to control the quality of the $\mathrm{x}$-ray pulse, and flat and linear beam phase space distributions perform better. Simulations indicate that EEHG pulses have less pedestal than SXRSS for $\leq 620 \mathrm{eV}$ assuming MBI growth is mitigated with the laser heater. Wakefields, especially CSR in the large dispersive section, have a large impact on spectral quality and favor longer beams. 
Overall, SXRSS has the obvious advantage of simplicity (assuming that adequate optics cooling can be provided at the desired high repetition rate of LCLS-II), but lacks some potential features for multipulse and multicolor FEL operations enabled by external laser seeding. Thus, these seeding options appear to be complementary, with SXRSS providing the strongest basic performance while EEHG allows for more specialized applications. Because the required physical infrastructures are noninterfering, the schemes can separately meet different expectations of user demand and FEL performance.

\section{ACKNOWLEDGMENTS}

The authors would like to thank E. Allaria, D. Cocco, J. Cryan, C. Feng, Y. Feng, L. Giannessi, A. Lutman, A. Marinelli, J. Qiang, E. Roussel, G. Stupakov, D. Xiang, and Z. Zhang for helpful conversations. This work was supported by the U.S. Department of Energy Contract No. DEAC02-76SF00515 and the U.S. DOE Office of Basic Energy Sciences under Award No. 2017-SLAC-100382.

[1] R. W. Schoenlein, New science opportunities enabled by Lcls-Ii X-Ray lasers, SLAC National Accelerator Laboratory, Report No. SLAC-R-1053, 2015.

[2] E. Hemsing, R. Coffee, G. Dakovski, W. M. Fawley, Y. Feng, G. Penn, J. Hastings, Z. Huang, G. Marcus, D. Ratner, T. Raubenheimer, R. W. Schoenlein, and Z. Zhang, Soft X-ray FEL seeding studies for LCLS-II:Task force status report, SLAC National Accelerator Laboratory, Report No. SLAC-TN-19-001, 2019.

[3] D. Ratner et al., Experimental Demonstration of a Soft X-Ray Self-Seeded Free-Electron Laser, Phys. Rev. Lett. 114, 054801 (2015).

[4] T. Kroll et al., X-ray absorption spectroscopy using a selfseeded soft X-ray free-electron laser, Opt. Express 24, 22469 (2016).

[5] P. Finetti et al., Pulse Duration of Seeded Free-Electron Lasers, Phys. Rev. X 7, 021043 (2017).

[6] M. B. Danailov, F. Bencivenga, F. Capotondi, F. Casolari, P. Cinquegrana, A. Demidovich, E. Giangrisostomi, M. P. Kiskinova, G. Kurdi, M. Manfredda, C. Masciovecchio, R. Mincigrucci, I. P. Nikolov, E. Pedersoli, E. Principi, and P. Sigalotti, Towards jitter-free pump-probe measurements at seeded free electron laser facilities, Opt. Express 22, 12869 (2014).

[7] E. Allaria et al., Two-stage seeded soft-X-ray free-electron laser, Nat. Photonics 7, 913 (2013).

[8] P. Rebernik Ribič et al., Coherent soft X-ray pulses from an echo-enabled harmonic generation free-electron laser, Nat. Photonics 13, 555 (2019).

[9] E. Allaria et al., The FERMI seeded-FEL facility: Status and perspectives, AIP Conf. Proc. 1741, 020006 (2016).

[10] L. Giannessi and C. Masciovecchio, FERMI: Present and future challenges, Appl. Sci. 7, 640 (2017).

[11] D. Gauthier, P. c. v. R. Ribič, G. De Ninno, E. Allaria, P. Cinquegrana, M. B. Danailov, A. Demidovich, E. Ferrari, and L. Giannessi, Generation of Phase-Locked Pulses from a Seeded Free-Electron Laser, Phys. Rev. Lett. 116, 024801 (2016).

[12] J. Amann et al., Demonstration of Self-Seeding in a HardX-Ray Free-Electron Laser, Nat. Photonics 6, 693 (2012).

[13] G. Stupakov, Using the Beam-Echo Effect for Generation of Short-Wavelength Radiation, Phys. Rev. Lett. 102, 074801 (2009).

[14] D. Xiang and G. Stupakov, Echo-enabled harmonic generation free electron laser, Phys. Rev. Accel. Beams 12, 030702 (2009).

[15] C. Feng, H. Deng, M. Zhang, X. Wang, S. Chen, T. Liu, K. Zhou, D. Gu, Z. Wang, Z. Jiang, X. Li, B. Wang, W. Zhang, T. Lan, L. Feng, B. Liu, Q. Gu, Y. Leng, L. Yin, D. Wang, Z. Zhao, G. Wang, and D. Xiang, Coherent extreme ultraviolet free-electron laser with echo-enabled harmonic generation, Phys. Rev. Accel. Beams 22, 050703 (2019).

[16] G. Penn, EEHG performance and scaling laws, LBNL Paper Report No. LBNL-6481E, 2014.

[17] E. Hemsing, M. Dunning, B. Garcia, C. Hast, T. Raubenheimer, G. Stupakov, and D. Xiang, Echo-enabled harmonics up to the 75 th order from precisely tailored electron beams, Nat. Photonics 10, 512 (2016).

[18] E. Hemsing, Minimum Spectral bandwidth in echo seeded free electron lasers, Front. Phys. 7, 35 (2019).

[19] F. Chao, H. Dazhang, D. Haixiao, C. Jianhui, X. Dao, L. Bo, W. Dong, and Z. Zhentang, A single stage EEHG at SXFEL for narrow-bandwidth soft X-ray generation, Science bulletin 61, 1202 (2016).

[20] J. MacArthur, J. Duris, Z. Huang, and A. Marinelli, in Proceedings, 8th International Particle Accelerator Conference (IPAC 2017): Copenhagen, Denmark, 2017 (JACoW, Geneva, Switzerland, 2017) p. WEPAB118.

[21] Z. Zhang, J. Duris, J. P. MacArthur, Z. Huang, and A. Marinelli, Double chirp-taper X-ray free-electron laser for attosecond pump-probe experiments, Phys. Rev. Accel. Beams 22, 050701 (2019).

[22] S. Reiche, GENESIS 1.3: a fully 3D time-dependent FEL simulation code, Nucl. Instrum. Methods Phys. Res., Sect. A 429, 243 (1999).

[23] J. Qiang, R. D. Ryne, M. Venturini, A. A. Zholents, and I. V. Pogorelov, High resolution simulation of beam dynamics in electron linacs for x-ray free electron lasers, Phys. Rev. ST Accel. Beams 12, 100702 (2009).

[24] We note that earlier studies on longer (150 fs core compared to 50-60 fs) $300 \mathrm{pC}$ e-beams showed inferior spectral performance for both SXRSS and for EEHG, particularly in terms of the fractional energy content of the pedestal [2].

[25] J. Feldhaus, E. L. Saldin, J. R. Schneider, E. A. Schneidmiller, and M. V. Yurkov, Possible application of $\mathrm{X}$-ray optical elements for reducing the spectral bandwidth of an X-ray SASE FEL, Opt. Commun. 140, 341 (1997).

[26] Y. Feng, J. Amann, D. Cocco, C. Field, J. Hastings, P. Heimann, Z. Huang, H. Loos, J. Welch, J., K. Chow, P. Emma, N. Rodes, and R. Schoenlein, in Proceedings of FEL 2012, Nara, Japan (2012), pp. 205-212, http:// accelconf.web.cern.ch/accelconf/FEL2012/papers/tuobi01 .pdf. 
[27] D. Cocco, R. Abela, J. W. Amann, K. Chow, P. J. Emma, Y. Feng, G. L. Gassner, J. Hastings, P. Heimann, Z. Huang, H. Loos, P. A. Montanez, D. Morton, H.-D. Nuhn, D. F. Ratner, L. N. Rodes, U. Flechsig, J. J. Welch, and J. Wu, in X-Ray Lasers and Coherent X-Ray Sources: Development and Applications $X$, Society of Photo-Optical Instrumentation Engineers (SPIE) Conference Series (2013), Vol. 8849, p. 88490A, https://doi.org/10.1117/ 12.2024402 .

[28] G. Marcus, W. M. Fawley, D. Bohler, Y. Ding, Y. Feng, E. Hemsing, Z. Huang, J. Krzywinski, A. Lutman, and D. Ratner, Experimental observations of seed growth and accompanying pedestal contamination in a self-seeded, soft x-ray free-electron laser, Phys. Rev. Accel. Beams 22, 080702 (2019).

[29] K. L. Bane and G. Stupakov, Resistive wall wakefield in the LCLS undulator beam pipe, Report No. SLAC-PUB10707, 2004, http://dx.doi.org/10.2172/833045.

[30] J. Krzywinski, R. Conley, S. Moeller, G. Gwalt, F. Siewert, C. Waberski, T. Zeschke, and D. Cocco, Damage thresholds for blaze diffraction gratings and grazing incidence optics at an X-ray free-electron laser, J. Synchrotron Radiat. 25, 85 (2018).

[31] Z. Zhang, G. Marcus, W. M. Fawley, E. Hemsing, Z. Huang, and A. Lutman, Statistical analysis of self-seeded $\mathrm{x}$-ray free-electron laser with microbunching instability (to be published).

[32] G. Stupakov, Effect of finite pulse length and laser frequency chirp on HGHG and EEHG seeding, Report No. SLAC-PUB-14639, 2011.

[33] D. Ratner, A. Fry, G. Stupakov, and W. White, Laser phase errors in seeded free electron lasers, Phys. Rev. Accel. Beams 15, 030702 (2012).

[34] D. Gauthier, P. c. v. R. Ribič, G. De Ninno, E. Allaria, P. Cinquegrana, M. B. Danailov, A. Demidovich, E. Ferrari, L. Giannessi, B. Mahieu, and G. Penco, Spectrotemporal Shaping of Seeded Free-Electron Laser Pulses, Phys. Rev. Lett. 115, 114801 (2015).

[35] G. Penn, B. Garcia, E. Hemsing, and G. Marcus, in Proceedings, 38th International Free Electron Laser Conference, FEL2017 (2018), p. MOP010, http:// accelconf.web.cern.ch/AccelConf/fel2017/papers/mop010 .pdf.

[36] G. De Ninno, B. Mahieu, E. Allaria, L. Giannessi, and S. Spampinati, Chirped Seeded Free-Electron Lasers: Self-Standing Light Sources for Two-Color Pump-Probe Experiments, Phys. Rev. Lett. 110, 064801 (2013).

[37] N. F. Scherer, R. J. Carlson, A. Matro, M. Du, A. J. Ruggiero, V. Romero-Rochin, J. A. Cina, G. R. Fleming, and S. A. Rice, Fluorescence-detected wave packet interferometry: Time resolved molecular spectroscopy with sequences of femtosecond phase-locked pulses, J. Chem. Phys. 95, 1487 (1991).

[38] J. A. Cina, Nonlinear wavepacket interferometry for polyatomic molecules, J. Chem. Phys. 113, 9488 (2000).
[39] D. Xiang and G. Stupakov, in Proceedings of the 23rd Particle Accelerator Conference, Vancouver, Canada, 2009 (IEEE, Piscataway, NJ, 2009), pp. 2367-2369, http://accelconf.web.cern.ch/AccelConf/PAC2009/papers/ we5rfp044.pdf.

[40] E. L. Saldin, E. A. Schneidmiller, and M. V. Yurkov, Study of a noise degradation of amplification process in a multistage HGHG FEL, Opt. Commun. 202, 169 (2002).

[41] G. Geloni, V. Kocharyan, and E. Saldin, Analytical studies of constraints on the performance for eehg fel seed lasers, arXiv:1111.1615.

[42] C. Feng, H. Deng, G. Wang, D. Wang, Z. Zhao, and D. Xiang, Slippage effect on energy modulation in seeded free-electron lasers with frequency chirped seed laser pulses, Phys. Rev. Accel. Beams 16, 060705 (2013).

[43] G. Wang, C. Feng, T. Zhang, D. Wang, and H. Deng, Study on the seed laser phase error multiplication in seeded free electron lasers, Nucl. Instrum. Methods Phys. Res., Sect. A 737, 237 (2014).

[44] At LCLS-II, enhanced slippage schemes such as modulating at an odd planar undulator harmonic are limited their applicability because of the larger $K$ values that increase MBI and ISR effects.

[45] E. Hemsing, Bunching phase and constraints on echo enabled harmonic generation, Phys. Rev. Accel. Beams 21, 050702 (2018).

[46] G. Geloni, E. Saldin, E. Schneidmiller, and M. Yurkov, Longitudinal impedance and wake from XFEL undulators. Impact on current-enhanced SASE schemes, Nucl. Instrum. Methods Phys. Res., Sect. A 583, 228 (2007).

[47] Z. Huang, Intrabeam Scattering in an X-ray FEL Driver, SLAC Report No. LCLS-TN-02-08, 2002.

[48] G. Dattoli and E. Sabia, Bunching coefficients in echoenabled harmonic generation, Phys. Rev. Accel. Beams 16, 070702 (2013).

[49] G. Stupakov, in Proceedings of FEL 2011, Shanghai, China (2011), pp. 44-52, http://accelconf.web.cern.ch/ AccelConf/FEL2011/papers/mopb20.pdf.

[50] N. Yampolsky and B. E. Carlsten, Beam debunching due to ISR-induced energy diffusion, Nucl. Instrum. Methods Phys. Res., Sect. A 870, 156 (2017).

[51] E. Hemsing et al., in Proceedings, 38th International Free Electron Laser Conference, FEL2017 (JACoW, Geneva, Switzerland, 2018), pp. 219-224, https:// doi.org/10.18429/JACoW-FEL2017-TUB01.

[52] A. Marinelli, R. Coffee, S. Vetter, P. Hering, G. N. West, S. Gilevich, A. A. Lutman, S. Li, T. Maxwell, J. Galayda, A. Fry, and Z. Huang, Optical Shaping of X-Ray Free-Electron Lasers, Phys. Rev. Lett. 116, 254801 (2016).

[53] A. A. Lutman, T. J. Maxwell, J. P. MacArthur, M. W. Guetg, N. Berrah, R. N. Coffee, Y. Ding, Z. Huang, A. Marinelli, S. Moeller, and J. C. U. Zemella, Fresh-slice multicolour X-ray free-electron lasers, Nat. Photonics $\mathbf{1 0}$, 745 (2016). 